\title{
Alterations of Interneurons of the Gerbil Hippocampus after Transient Cerebral Ischemia: Effect of Pitavastatin
}

\author{
Toshiki Himeda', Natsumi Hayakawa', Hiroko Tounai', Mio Sakuma', Hiroyuki Kato² and Tsutomu Araki*,', \\ 'Department of Drug Metabolism and Therapeutics, Graduate School and Faculty of Pharmaceutical Sciences, The University of Tokushima, \\ Tokushima, Japan; ${ }^{2}$ Department of Neurology, Organized Center of Clinical Medicine, International University of Health and Welfare, \\ Tochigi, Japan
}

\begin{abstract}
We investigated the immunohistochemical alterations of parvalbumin (PV)-expressing interneurons in the hippocampus after transient cerebral ischemia in gerbils in comparison with neuronal nitric oxide synthase (nNOS)-expressing interneurons. We also examined the effect of 3-hydroxy-3-methylglutaryl-coenzyme A reductase inhibitor pitavastatin against the damage of neurons and interneurons in the hippocampus after cerebral ischemia. Severe neuronal damage was observed in the hippocampal CAI pyramidal neurons 5 and I 4 days after ischemia. The PV immunoreactivity was unchanged up to 2 days after ischemia. At 5 and 14 days after ischemia, in contrast, a conspicuous reduction of PV immunoreactivity was observed in interneurons of the hippocampal CAI sector. Furthermore, a significant decrease of PV immunoreactivity was found in interneurons of the hippocampal CA3 sector. No damage of nNOS-immunopositive interneurons was detected in the gerbil hippocampus up to I day after ischemia. Thereafter, a decrease of nNOS immunoreactive interneurons was found in the hippocampal CAI sector up to I 4 days after ischemia. Pitavastatin significantly prevented the neuronal cell loss in the hippocampal CAI sector 5 days after ischemia. Our immunohistochemical study also showed that pitavastatin prevented significant decrease of PV- and nNOS-positive interneurons in the hippocampus after ischemia. Double-labeled immunostainings showed that PV immunoreactivity was not found in nNOS-immunopositive interneurons of the brain. The present study demonstrates that cerebral ischemia can cause a loss of both PV- and nNOS-immunoreactive interneurons in the hippocampal CAI sector. Our findings also show that the damage to nNOS-immunopositive interneurons may precede the neuronal cell loss in the hippocampal CAI sector after ischemia and nNOS-positive interneurons may play some role in the pathogenesis of cerebral ischemic diseases. Furthermore, our present study indicates that pitavastatin can prevent the damage of interneurons in the hippocampus after cerebral ischemia. Thus, our study provides valuable information for the pathogenesis after cerebral ischemia.

Neuropsychopharmacology (2005) 30, 20I4-2025. doi:I 0. I038/sj.npp. I 300798; published online 22 June 2005
\end{abstract}

Keywords: interneurons; HMG-CoA reductase; immunohistochemistry; parvalbumin; nNOS; gerbil

\section{INTRODUCTION}

Selective vulnerability of neurons in the central nervous system (CNS) to a brief period of transient cerebral ischemia is a well-known phenomenon that is particularly evidence in the hippocampus (Kirino, 1982; Pulsinelli et al, 1982; Araki et al, 1992, 1993). The pyramidal neurons in the hippocampal CA1 sector begin to degenerate a few days after ischemia. In contrast, interneurons of the hippocampal CA1 sector and granule cells of dentate gyrus are resistant to

\footnotetext{
* Correspondence: Professor T Araki, Department of Drug Metabolism and Therapeutics, Graduate School and Faculty of Pharmaceutical Sciences, The University of Tokushima, 1-78, Sho-machi, Tokushima 770-8505, Japan, Tel: + 8188633 7277, Fax: +8I 88633 95।I, E-mail: tsuaraki@ph.tokushima-u.ac.jp

Received 8 February 2005; revised 12 April 2005; accepted 28 April 2005

Online publication: I I May 2005 at http://www.acnp.org/citations/ Npp05 I005050079/default.pdf
}

such a brief period of ischemic insult (Nitsch et al, 1989; Tortosa and Ferrer, 1993). A similar pattern is seen in the human hippocampus after cerebral ischemia (Petito et al, 1987). A previous study has demonstrated that nonpyramidal neurons, like interneurons, in the hippocampal CA1 sector, can survive transient ischemia, but undergo degenerative changes following complete loss of CA1 pyramidal neurons (Fukuda et al, 1993). This observation suggests that interneurons are not resistant to prolonged periods of cerebral ischemia. Therefore, the postischemic changes of interneurons in the hippocampal CA1 sector which is most vulnerable to ischemia are not fully understood.

Calcium-binding proteins such as parvalbumin (PV) are thought to protect neurons against cytotoxic calcium overload because of their potential buffering ability (Tortosa and Ferrer, 1993; Ferrer et al, 1998). Parvalbumin is a cytosolic calcium buffer expressed in GABAergic $(\gamma$-aminobutyrate) interneurons and is present numerous 
nuclei of the brain (Andressen et al, 1993; Hontanilla et al, 1998). Therefore, it is well known that PV is a superior marker for a subpopulation of GABAergic interneurons (Celio, 1986).

A previous study suggests that 3-hydroxy-3-methylglutaryl-coenzyme A (HMG-CoA) reductase inhibitors decrease cerebral ischemia and infarct size in normocholesterolemic mice (Endres et al, 1998). A recent interesting study suggests that HMG-CoA reductase inhibitor inhibits the formation of TNF- $\alpha$ and peroxynitrite in activated microglia, thereby protecting dopaminergic neurons from inflammatory damage (Selley, 2005). Pitavastatin (NK-104) is a novel synthetic potent and selective inhibitor of HMGCoA reductase, the rate-limiting enzyme in cholesterol biosynthesis (Aoki et al, 1997). This compound is known to lower plasma total cholesterol levels and reduce triglyceride levels (Kajinami et al, 2000). We recently reported that pitavastatin can protect against the hippocampal CA1 neuronal damage after transient cerebral ischemia in gerbils (Kumagai et al, 2004; Muramatsu et al, 2004). These observations indicate that the HMG-CoA reductase inhibitor pitavastatin is effective against transient cerebral ischemia. However, little is known about the effect of pitavastatin against changes of interneurons in the hippocampal CA1 sector after transient cerebral ischemia.

In the present study, therefore, we investigated the immunohistochemical alterations of PV-positive interneurons in the hippocampus after $5 \mathrm{~min}$ of transient cerebral ischemia in gerbils in comparison with neuronal nitric oxide (NO) synthase (nNOS)-positive cells, which are limited to interneurons in the hippocampus. We also examined the effect of HMG-CoA reductase inhibitor pitavastatin against the changes of interneurons in the hippocampal CA1 sector after cerebral ischemia.

\section{MATERIALS AND METHODS}

\section{Experimental Animals}

Male adult Mongolian gerbils, weighing 60-90 g (Nihon SLC Co., Shizuoka, Japan), were used. The animals were housed in a controlled environment $\left(23 \pm 1^{\circ} \mathrm{C}, 50 \pm 5 \%\right.$ humidity) and were allowed food and tap water ad libitum. The room lights were on between 0800 and $2000 \mathrm{~h}$. The animals were anesthetized with $2 \%$ halothane in a mixture of $30 \%$ oxygen and $70 \%$ nitrous oxides. Bilateral carotid arteries were exposed and anesthesia was discontinued to minimize its effect. The carotid arteries were clamped with aneurismal clips for $5 \mathrm{~min}$, and then the animals were allowed to survive for $1 \mathrm{~h}(n=6), 5 \mathrm{~h}(n=5), 1$ day $(n=5), 2$ days $(n=5)$, 5 days $(n=8)$, and 14 days $(n=5)$ after ischemia. Shamoperated animals $(n=7)$ were treated in the same manner, except for the clipping of the bilateral carotid arteries. Body temperature was maintained at $37-38^{\circ} \mathrm{C}$ with a heating pad equipped with a thermostat until the animals started moving. All experiments were performed in accordance with the Guidelines for Animal Experiments of the Tokushima University School of Medicine.

\section{Drug Treatments}

To evaluate the neuroprotective effect of drug treatment, pitavastatin at doses of 3,10 , or $30 \mathrm{mg} / \mathrm{kg}$ was administrated orally twice a day for 5 days before transient cerebral ischemia. Transient cerebral ischemia was induced in gerbils $1 \mathrm{~h}$ after the last treatment with pitavastatin. Vehicle ( $0.5 \%$ carboxymethylcellulose (CMC)) was also administered in sham-operated gerbils under the same conditions. Pitavastatin was generously provided by Kowa company, Ltd, Tokyo, Japan.

\section{Immunohistochemistry}

The animals were anesthetized with sodium pentobarbital ( $50 \mathrm{mg} / \mathrm{kg}$, i.p.) $1,5 \mathrm{~h}, 1,2,5$, and 14 days after cerebral ischemia, and the brains were perfusion-fixed with $4 \%$ paraformaldehyde in $0.1 \mathrm{M}$ phosphate buffer ( $\mathrm{pH} 7.4$ ) following a heparinized saline flush. The brains were removed $1 \mathrm{~h}$ after perfusion fixation at $4^{\circ} \mathrm{C}$ and were immersed in the same fixative until they were embedded in paraffin. Paraffin sections, $5 \mu \mathrm{m}$ in thickness, were stained with cresyl violet and used for immunohistochemistry.

For immunohistochemical studies, a Vectastain elite ABC kit (Vector Labs., Burlingame, CA, USA), mouse anti-PV monoclonal antibody (Chemicon International, Inc., Temecula, CA, USA), and rabbit anti-nNOS polyclonal antibody (Zymed Labs., San Francisco, CA, USA) were used. The immunohistochemical staining with anti-PV antibody $(1: 500)$ and anti-nNOS antibody $(1: 500)$ was performed as described previously (Kurosaki et al, 2002; Muramatsu et al, 2002). Negative control sections were treated in the same way, except that each antibody was omitted. Immunoreactions for $\mathrm{PV}$ and nNOS staining were visualized using Vector DAB $\left(3^{\prime}, 3^{\prime}\right.$-diaminobenzidine) substrate kit (Vector Labs., Burlingame, CA, USA) and Vector NovaRed substrate kit (Vector Labs., Burlingame, CA, USA).

The immunostaining for PV and nNOS was semiquantitatively graded as intense (grade 3 ), moderate (grade 2), weak (grade 1), and not detectable (grade 0 ), without the examiner knowing the experimental protocols, as described previously (Kato et al, 1995; Muramatsu et al, 2003; Kurosaki et al, 2005). Values were expressed means \pm SD. Statistical significance was evaluated using the nonparametric Dunnett's multiple comparison test.

Double-labeled immunostaining with anti-PV and antinNOS antibodies was performed in some sections of gerbils. The immunohistochemical staining with anti-NOS antibody was performed as described above. Then, the double-labeled immunostaining with anti-PV antibody was performed as described above. Immunoreactions for PV and nNOS staining were visualized using Vector DAB substrate kit (brown) and Vector NovaRed substrate kit (red), respectively.

\section{RESULTS}

\section{Neuronal Loss in the Hippocampus after Transient Cerebral Ischemia}

Neuronal death in CA1 pyramidal cells after ischemia was elucidated by cresyl violet staining. In the hippocampus, sham-operated gerbils showed no neuronal damage throughout the experiments. Gerbils subjected to ischemia showed no neuronal damage in the hippocampus up to 2 days after ischemia. At 5 and 14 days after ischemia, severe 
neuronal damage was observed in the hippocampal CA1 pyramidal neurons. However, the neuronal damage was not seen in the hippocampal CA3 sector and dentate gyrus. Prophylactic treatment with pitavastatin significantly prevented the neuronal cell loss in the hippocampal CA1 sector 5 days after ischemia, as shown in Figures 1 and 2.

\section{PV Staining in the Hippocampus after Transient Cerebral Ischemia}

Representative microphotographs of $\mathrm{PV}$ staining in the hippocampus are shown in Figures 3 and 4. In shamoperated gerbils, PV-immunoreactive interneurons were clearly evident in the hippocampus. The majority of PVimmunoreactive cell bodies with varicose dendrites were located to the hippocampus. In animals subjected to ischemia, the PV immunoreactivity was unchanged up to 2 days after ischemia. At 5 and 14 days after ischemia, in contrast, a conspicuous reduction of PV immunoreactivity was noted in interneurons of the hippocampal CA1 sector. Furthermore, a significant decrease of PV immunoreactivity was found in interneurons of the hippocampal CA3 sector. However, dentate gyrus showed no significant changes throughout the experiments. In contrast, prophylactic treatment with pitavastatin significantly prevented a loss of PV-immunoreactive interneurons in the hippocampal CA1 sector 5 days after ischemia, as shown in Tables 1 and 2. However, prophylactic treatment with pitavastatin did not prevent the reduction of $\mathrm{PV}$-immunoreactive interneurons in the hippocampal CA3 sector. Furthermore, this compound showed no significant changes of PV-immunoreactive cells in dentate gyrus 5 days after ischemia.

\section{nNOS Staining in the Hippocampus after Transient Cerebral Ischemia}

Representative microphotographs of nNOS staining in the hippocampus are shown in Figures 5 and 6 . nNOS immunoreactivity was observed in interneurons of the hippocampus in sham-operated gerbils. No damage of nNOS-immunopositive interneurons was detected in the gerbil hippocampus up to 1 day after transient forebrain ischemia. Thereafter, a decrease of nNOS immunoreactivity was found in the hippocampal CA1 sector 2, 5, and 14 days after ischemia. In the hippocampal CA3 sector, nNOS immunoreactivity decreased only 5 days after ischemia. In contrast, the changes of nNOS immunoreactivity were not observed in the granule cells throughout the experiments, except for a transient decrease in this region 2 days after ischemia. Prophylactic treatment with pitavastatin significantly prevented a loss of nNOS-immunoreactive interneurons in the hippocampal CA1 and CA3 sector 5 days after ischemia. Furthermore, this compound showed no significant changes of nNOS-immunoreactive cells in the dentate gyrus 5 days after ischemia, as shown in Tables 1 and 2 .

\section{Double-Labeled Immunostainings}

Representative microphotographs of double-labeled immunostainings with anti-PV and anti-nNOS antibody are shown in Figure 7. PV immunoreactivity was not found in
nNOS-immunopositive interneurons of the hippocampus and cerebral cortex. Thus, the interneuron with PV was different from that with nNOS.

\section{DISCUSSION}

It is well known that calcium plays a key role in ischemic neuronal damage (Paschen, 1996). Calcium-binding proteins belonging to the EF-hand family function as highaffinity, selective intracellular calcium buffers. One of these calcium-binding proteins, $\mathrm{PV}$, is present throughout the brain, where it is selectively localized in a subpopulation of GABA-containing neurons (Heizmann, 1984; Celio, 1986). Therefore, the PV has been regarded as a superior marker for a subpopulation of GABAergic interneurons throughout the brain (Celio, 1986; Schwartz-Bloom and Sah, 2001). A previous study has shown that $\mathrm{PV}$-immunoreactive neurons are particularly resistant to transient ischemic insult which produces hippocampal CA1 neuronal death (Nitsch et al, 1989). Furthermore, Tortosa and Ferrer (1993) also demonstrated that PV-immunoreactive neurons in the hippocampus are preserved after a brief period of cerebral ischemia in gerbils. For this reason, it is suggested that the calcium-buffering properties of PV protect the GABAergic neurons from ischemic cell damage. PV-containing neurons also exhibit a higher survival rate in the human epileptic hippocampus (Sloviter et al, 1991), and, recently, a protective effect of PV on excitotoxic motor neurons death has been observed (Van Den Bosch et al, 2002). On the other hand, other studies suggest that PV-immunoreactive neurons were gradually depleted in the brain of gerbils after a longer period of ischemia (Fukuda et al, 1993). Furthermore, PV-containing neurons are supposed to be selectively vulnerable in Alzheimer's disease (Solodkin et al, 1996) and not to be protected in some forms of epilepsy (Bouilleret et al, 2000). Thus, much evidence for the vulnerability and role of PV-containing neurons to stress response such as ischemia and epilepsy is contradictory.

Increasing evidence demonstrates that NO participates in the mechanisms of cerebral ischemia and much attention has been focused on the role of NO in the pathogenesis of ischemic brain damage. Excessive production of $\mathrm{NO}$, and consequent increase of the oxygen-free radical peroxynitrite, has been implicated in neuronal cell loss occurring both in acute and chronic brain pathologies (Dawson and Dawson, 1995; Iadecola, 1997; Yun et al, 1997). However, the precise role of $\mathrm{NO}$ in the pathogenesis of ischemic brain damage is not fully understood (Choi, 1993; Iadecola et al, 1994). NO, a free radical gas, synthesized from L-arginine by NOS is involved in a wide range of physiological and pathological mechanisms, including the control of the cardiovascular, immune, and nervous systems, as well as cellular neurotoxicity (Dawson et al, 1991; Moncada et al, 1991; Dawson and Snyder, 1994). Three isoforms of NOS enzymes are traditionally recognized: nNOS, inducible NOS (iNOS), and endothelial NOS (eNOS). Among the three isoforms of NOS enzymes, nNOS is the main NOS isoform in the brain, as its catalytic activity and proteins are identifiable throughout the CNS (Bredt et al, 1991; Huang et al, 1993). It is known that nNOS is restricted to occasional 

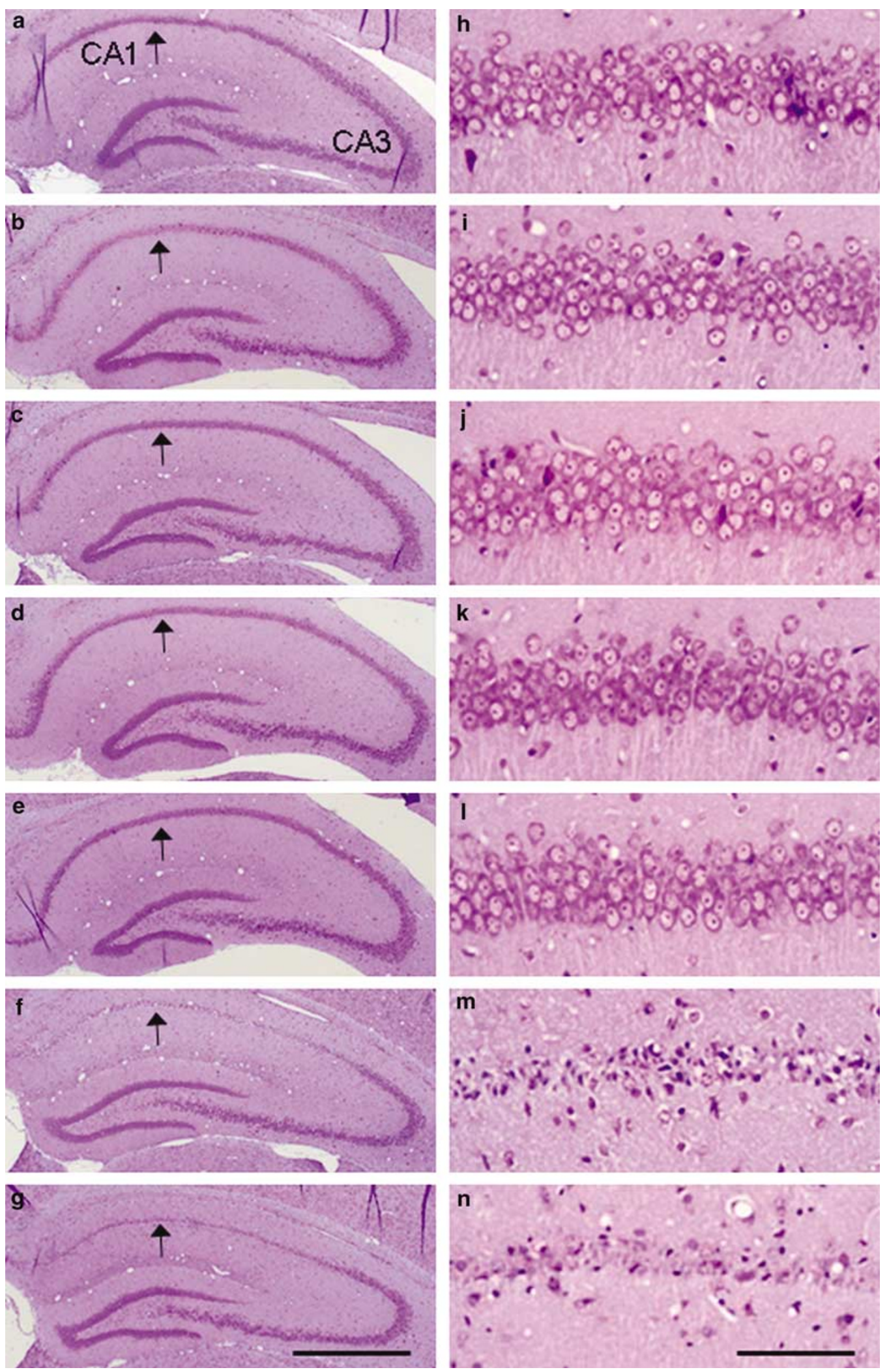

Figure I Representative photographs with Cresyl violet staining in the hippocampus (a-g) and CAI sector (h-n) after transient cerebral ischemia. $(\mathrm{a}, \mathrm{h})$ : sham-operated gerbil brain. (b, i): vehicle-treated gerbil brain I h after ischemia. (c, j): vehicle-treated gerbil brain $5 \mathrm{~h}$ after ischemia. (d, k): vehicletreated gerbil brain I day after ischemia. (e, l): vehicle-treated gerbil brain 2 days after ischemia. (f, $\mathrm{m}$ ): vehicle-treated gerbil brain 5 days after ischemia. $(\mathrm{g}, \mathrm{n})$ : vehicle-treated gerbil brain 14 days after ischemia. Arrows show location of changes. Each group contained 5-8 animals. Bar $(\mathrm{a}-\mathrm{g})=\mathrm{I} \mathrm{mm}$. $\operatorname{Bar}(\mathrm{h}-\mathrm{n})=100 \mu \mathrm{m}$. 



Figure 2 Effect of pitavastatin on neuronal densities in the CAI sector $\left(\mathrm{a}^{\prime}-\mathrm{e}^{\prime}\right)$ of the hippocampus (a-e) of the gerbil brain 5 days after transient cerebral ischemia. (a, $\left.\mathrm{a}^{\prime}\right)$ : sham-operated gerbil brain. (b, $\left.b^{\prime}\right)$ : vehicle-treated gerbil brain 5 days after ischemia. (c, $\left.c^{\prime}\right)$ : pitavastatin ( $3 \mathrm{mg} / \mathrm{kg}$ )-treated gerbil brain 5 days after ischemia. $\left(d, d^{\prime}\right)$ : pitavastatin $\left(10 \mathrm{mg} / \mathrm{kg}\right.$ )-treated gerbil brain 5 days after ischemia. (e, $\left.\mathrm{e}^{\prime}\right)$ : pitavastatin $(30 \mathrm{mg} / \mathrm{kg})$-treated gerbil brain 5 days after ischemia. Arrows show location of changes. Each group contained 7-8 animals. Bar $(\mathrm{a}-\mathrm{e})=1 \mathrm{~mm}$. Bar $\left(\mathrm{a}^{\prime}-\mathrm{e}^{\prime}\right)=100 \mu \mathrm{m}$.

interneurons in the hippocampus (Dinerman et al, 1994). In the mouse striatum, furthermore, nNOS-positive cells are known to be interneurons (Zucker et al, 2005). However, little is known about the exact changes of nNOS expression as interneurons in the hippocampus after transient cerebral ischemia in gerbils.

In the present study, no neuronal damage in the hippocampus was observed up to 2 days after transient cerebral ischemia. At 5 and 14 days after ischemia, severe neuronal damage was found only in the hippocampal CA1 sector. The PV immunoreactivity was also unchanged up to 2 days after ischemia. At 5 and 14 days after ischemia, however, a conspicuous reduction of $\mathrm{PV}$ immunoreactivity was noted in interneurons of the hippocampal CA1 sector. Furthermore, a significant decrease of PV immunoreactivity was found in interneurons of the hippocampal CA3 sector. 

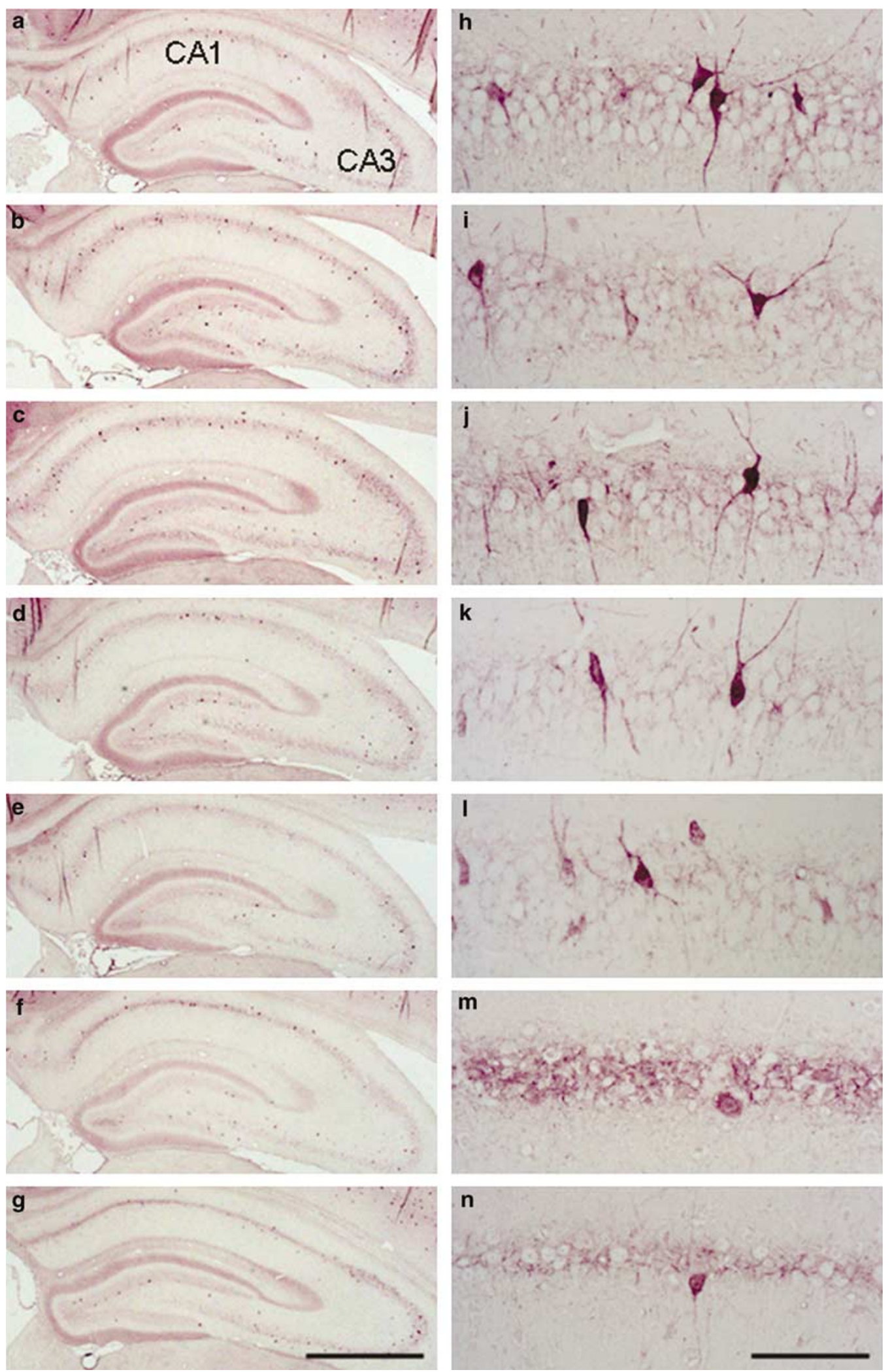

Figure 3 Representative photomicrographs with PV immunostaining of the hippocampus (a-g) and CAI sector (h-n) after transient cerebral ischemia. $(\mathrm{a}, \mathrm{h})$ : sham-operated gerbil brain. (b, i): vehicle-treated gerbil brain I h after ischemia. (c, j): vehicle-treated gerbil brain $5 \mathrm{~h}$ after ischemia. (d, k): vehicletreated gerbil brain I day after ischemia. (e, l): vehicle-treated gerbil brain 2 days after ischemia. (f, m): vehicle-treated gerbil brain 5 days after ischemia. $(g, n)$ : vehicle-treated gerbil brain 14 days after ischemia. Each group contained 5-8 animals. Bar $(a-g)=1 \mathrm{~mm}$. Bar $(\mathrm{h}-\mathrm{n})=100 \mu \mathrm{m}$. 

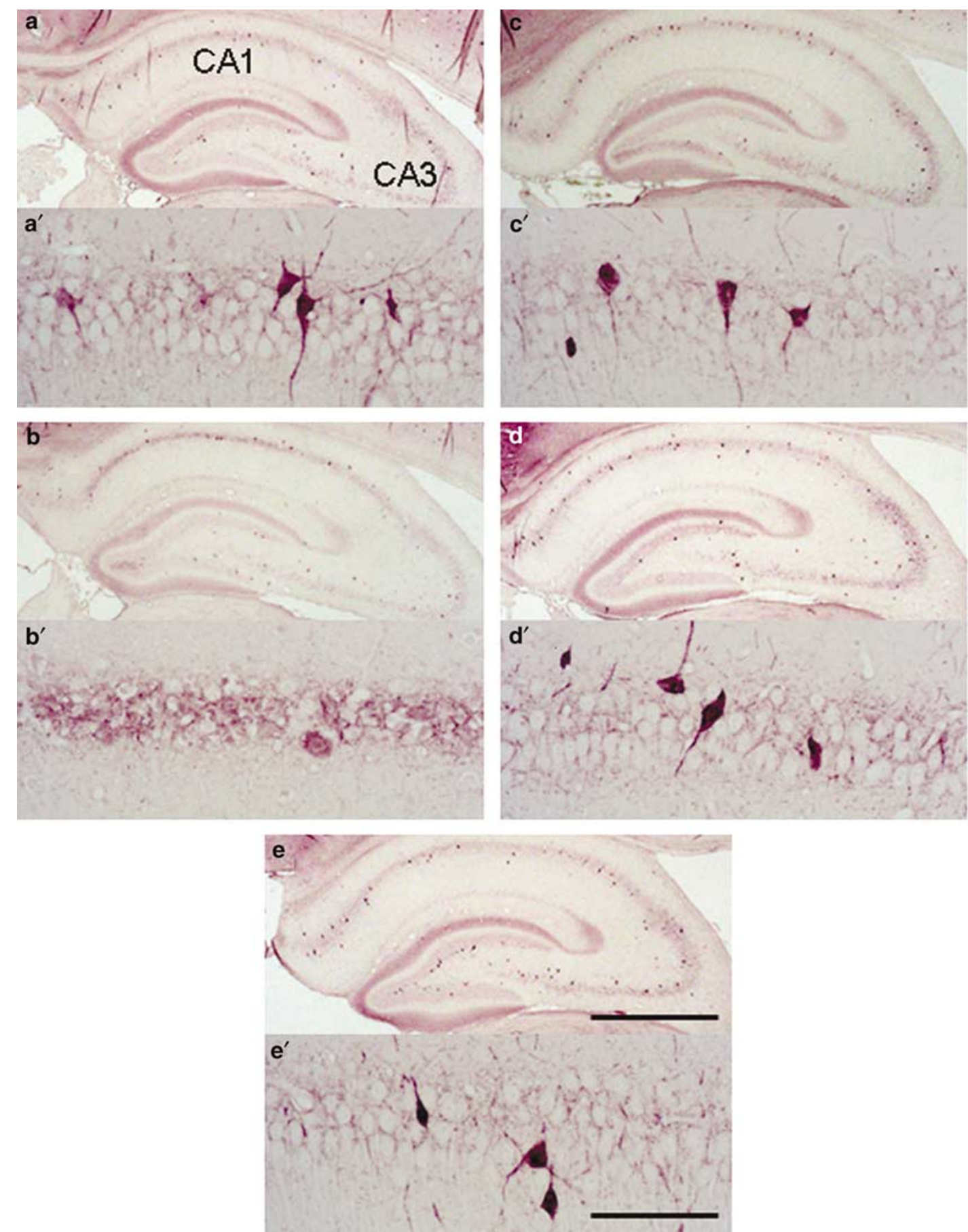

Figure 4 Effect of pitavastatin on PV immunoreactivity in the CAI sector ( $\left.\mathrm{a}^{\prime}-\mathrm{e}^{\prime}\right)$ of the hippocampus (a-e) of the gerbil brain 5 days after transient cerebral ischemia. (a, $\left.\mathrm{a}^{\prime}\right)$ : sham-operated gerbil brain. $\left(b, b^{\prime}\right)$ : vehicle-treated gerbil brain 5 days after ischemia. (c, $\left.c^{\prime}\right)$ : pitavastatin ( $\left.3 \mathrm{mg} / \mathrm{kg}\right)$-treated gerbil brain 5 days after ischemia. (d, d'): pitavastatin $\left(10 \mathrm{mg} / \mathrm{kg}\right.$ )-treated gerbil brain 5 days after ischemia. (e, e $\left.\mathrm{e}^{\prime}\right)$ : pitavastatin ( $\left.30 \mathrm{mg} / \mathrm{kg}\right)$-treated gerbil brain 5 days after ischemia. Each group contained 7-8 animals. $\operatorname{Bar}(\mathrm{a}-\mathrm{e})=1 \mathrm{~mm}$. Bar $\left(\mathrm{a}^{\prime}-\mathrm{e}^{\prime}\right)=100 \mu \mathrm{m}$.

However, the dentate gyrus showed no significant changes throughout the experiments. On the other hand, no damage of nNOS-immunopositive interneurons was detected in the gerbil hippocampus up to 1 day after transient cerebral ischemia. Thereafter, a decrease of nNOS immunoreactivity was found in the hippocampal CA1 sector at 2, 5, and 14 days after ischemia. In the hippocampal CA3 sector, nNOS immunoreactivity decreased only 5 days after ischemia. In contrast, the changes of nNOS immunoreactivity were not observed in the granule cells throughout the experiments, except for a transient decrease in this region 2 days after ischemia. These results indicate that transient cerebral ischemia can cause a loss of both PV and nNOSimmunoreactive interneurons in the hippocampal CA1 sector. Furthermore, our findings suggest that the damage to nNOS-immunopositive interneurons may precede the 
Table I Time Course of Parvalbumin and nNOS Immunoreactivity in the Gerbil Hippocampus after Transient Cerebral Ischemia

\begin{tabular}{|c|c|c|c|}
\hline & CAI sector & CA3 sector & Dentate gyrus \\
\hline \multicolumn{4}{|l|}{ Parvalbumin } \\
\hline Sham & $2.0 \pm 0.3$ & $2.3 \pm 0.4$ & $1.8 \pm 0.4$ \\
\hline I h & $2.0 \pm 0.6$ & $2.2 \pm 0.5$ & $1.8 \pm 0.4$ \\
\hline $5 \mathrm{~h}$ & $2.7 \pm 0.3$ & $2.4 \pm 0.3$ & $1.9 \pm 0.2$ \\
\hline I day & $2.1 \pm 0.3$ & $2.1 \pm 0.1$ & $1.9 \pm 0.4$ \\
\hline 2 days & $1.7 \pm 0.4$ & $1.9 \pm 0.3$ & $1.6 \pm 0.5$ \\
\hline 5 days & $0.9 \pm 0.5 *$ & $1.4 \pm 0.3 * *$ & $1.4 \pm 0.2$ \\
\hline 14 days & $0.7 \pm 0.3 * *$ & $1.5 \pm 0.2 *$ & $1.8 \pm 0.3$ \\
\hline \multicolumn{4}{|l|}{ nNOS } \\
\hline Sham & $2.6 \pm 0.3$ & $2.9 \pm 0.2$ & $2.4 \pm 0.4$ \\
\hline I h & $2.4 \pm 0.2$ & $2.9 \pm 0.2$ & $2.3 \pm 0.3$ \\
\hline $5 \mathrm{~h}$ & $2.8 \pm 0.2$ & $2.9 \pm 0.2$ & $2.0 \pm 0.4$ \\
\hline I day & $2.8 \pm 0.3$ & $2.8 \pm 0.3$ & $2.1 \pm 0.4$ \\
\hline 2 days & $1.8 \pm 0.4 *$ & $2.5 \pm 0.4$ & $1.7 \pm 0.5^{*}$ \\
\hline 5 days & $1.5 \pm 0.4 * * *$ & $2.4 \pm 0.3 *$ & $2.0 \pm 0.6$ \\
\hline 14 days & $1.6 \pm 0.6 *$ & $2.6 \pm 0.5$ & $2.4 \pm 0.4$ \\
\hline
\end{tabular}

The immunoreactivity was semiquantitatively graded as intense (grade 3), moderate (grade 2), weak (grade I) and not detectable (grade 0). Values were expressed as mean \pm SD. Each group contained 5-8 animals. Statistical significance was evaluated by nonparametric Dunnett's multiple comparison test ( $p<0.05$, ** $p<0.01$ compared with the sham-operated control group).

Table 2 Effect of Pitavastatin on Parvalbumin and nNOS Immunoreactivity in the Gerbil Hippocampus 5 days after Transient Cerebral Ischemia

\begin{tabular}{|c|c|c|c|}
\hline & CAI sector & CA3 sector & Dentate gyrus \\
\hline \multicolumn{4}{|l|}{ Parvalbumin } \\
\hline Sham & $2.0 \pm 0.3 * *$ & $2.3 \pm 0.4 * *$ & $1.8 \pm 0.4$ \\
\hline Ischemia $+0.5 \%$ CMC & $1.0 \pm 0.5$ & $1.4 \pm 0.3$ & $1.4 \pm 0.2$ \\
\hline \multicolumn{4}{|l|}{ Ischemia+Pitavastatin } \\
\hline Pitavastatin (3 mg/kg) & $1.5 \pm 0.5$ & $1.4 \pm 0.3$ & $1.3 \pm 0.2$ \\
\hline Pitavastatin $(10 \mathrm{mg} / \mathrm{kg})$ & $1.8 \pm 0.6 *$ & $1.8 \pm 0.4$ & $1.6 \pm 0.5$ \\
\hline Pitavastatin $(30 \mathrm{mg} / \mathrm{kg})$ & $1.9 \pm 0.2 * *$ & $1.8 \pm 0.2$ & $1.7 \pm 0.2$ \\
\hline \multicolumn{4}{|l|}{ nNOS } \\
\hline Sham & $2.6 \pm 0.3 * * *$ & $2.9 \pm 0.2 * * *$ & $2.4 \pm 0.4$ \\
\hline Ischemia $+0.5 \%$ CMC & $1.5 \pm 0.4$ & $2.4 \pm 0.4$ & $2.0 \pm 0.6$ \\
\hline \multicolumn{4}{|l|}{ Ischemia+Pitavastatin } \\
\hline Pitavastatin (3 mg/kg) & $2.1 \pm 0.5$ & $2.7 \pm 0.4$ & $2.0 \pm 0.3$ \\
\hline Pitavastatin $(10 \mathrm{mg} / \mathrm{kg})$ & $2.4 \pm 0.4 *$ & $2.8 \pm 0.3^{*}$ & $2.3 \pm 0.4$ \\
\hline Pitavastatin $(30 \mathrm{mg} / \mathrm{kg})$ & $2.3 \pm 0.7 *$ & $2.8 \pm 0.2 *$ & $2.1 \pm 0.6$ \\
\hline
\end{tabular}

The immunoreactivity was semiquantitatively graded as intense (grade 3), moderate (grade 2), weak (grade I) and not detectable (grade 0). Values were expressed as mean \pm SD. Each group contained 7-8 animals. Statistical significance was evaluated by nonparametric Dunnett's multiple comparison test ( $p<0.05, * * * 0.01$ compared with ischemia $+0.5 \%$ CMC group). neuronal cell loss in the hippocampal CA1 sector after ischemia.

The present study with our double-labeled immunostainings demonstrates that the interneuron with PV was different from that with nNOS. nNOS has been shown to account for $95 \%$ or more of all NOS catalytic activity in the brain under basal conditions (Huang et al, 1993). Immunohistochemical studies have shown that $1-2 \%$ of neurons in the cortex contain NOS immunoreactivity (Bredt et al, 1991), later equated with neuronal isoform. These neurons constitute a relatively small subpopulation of interneurons, which also contain GABA, neuropeptide $\mathrm{Y}$, and somatostatin (Vincent and Kimura, 1992; Valtschanoff et al, 1993). However, PV has also been regarded as a superior marker for a subpopulation of GABAergic interneurons throughout the brain (Celio, 1986). In the present study, therefore, the exact reason that PV-immunoreactive cells and nNOS-immunoreactive cells were clearly different interneurons in both cerebral cortex and hippocampal CA1 sector of gerbils is presently unclear. In this respect, a previous study demonstrated that double labeling of $\mathrm{nNOS}$ with calretinin or with PV did not show any double-labeled cells in the monkey cerebral cortex (Smiley et al, 2000). We recently reported that $\mathrm{PV}$-immunoreactive cells and nNOSimmunoreactive cells were clearly different interneurons in both the striatum and substantia nigra of mice (Kurosaki et al, 2005). Therefore, we speculate that PV-immunoreactive interneurons and nNOS-immunoreactive interneurons may play a different role in the brain, although further studies should be performed to investigate the precise mechanism responsible for our findings.

Of particular interest in the present study is that the damage to nNOS-immunopositive interneurons preceded the neuronal cell loss in the hippocampal CA1 sector after ischemia. Although the reason for this phenomenon is presently unclear, we speculate that nNOS interneurons may play some role in the pathogenesis of cerebral ischemic diseases. Furthermore, a significant loss of PV immunoreactivity and nNOS immunoreactivity was found in interneurons of the hippocampal CA3 sector. For this reason, we suggest that transient cerebral ischemia may cause the functional damage of interneurons in the hippocampal CA3 sector. However, the precise mechanisms responsible for such findings should be investigated in further studies.

Pitavastatin is a novel potent HMG-CoA reductase inhibitor with prolonged effect and has been approved for treatment of hyperlipoproteinemia (Aoki et al, 1997; Fujimoto et al, 1999). This drug is also known to lower plasma total cholesterol levels and reduce triglyceride levels. Furthermore, pitavastatin processes a 10-fold higher cholesterol-lowering effect, compared with other HMGCoA reductase inhibitors such as pravastatin and simvastatin (Aoki et al, 1997; Suzuki et al, 1999). A recent study indicates that long-term treatment with pitavastatin is effective and safe for patients with heterozygous familial hypercholesterolemia (Noji et al, 2002). From these observations, it is suggested that pitavastatin is a new and potentially superior therapeutic agent in comparison with other currently available statins. Several studies suggest that HMG-CoA reductase inhibitors can affect expression of eNOS (endothelial NO synthase), increasing the bioavailability of NO (Laufs et al, 1997; John et al, 1998; Kaesemeyer 

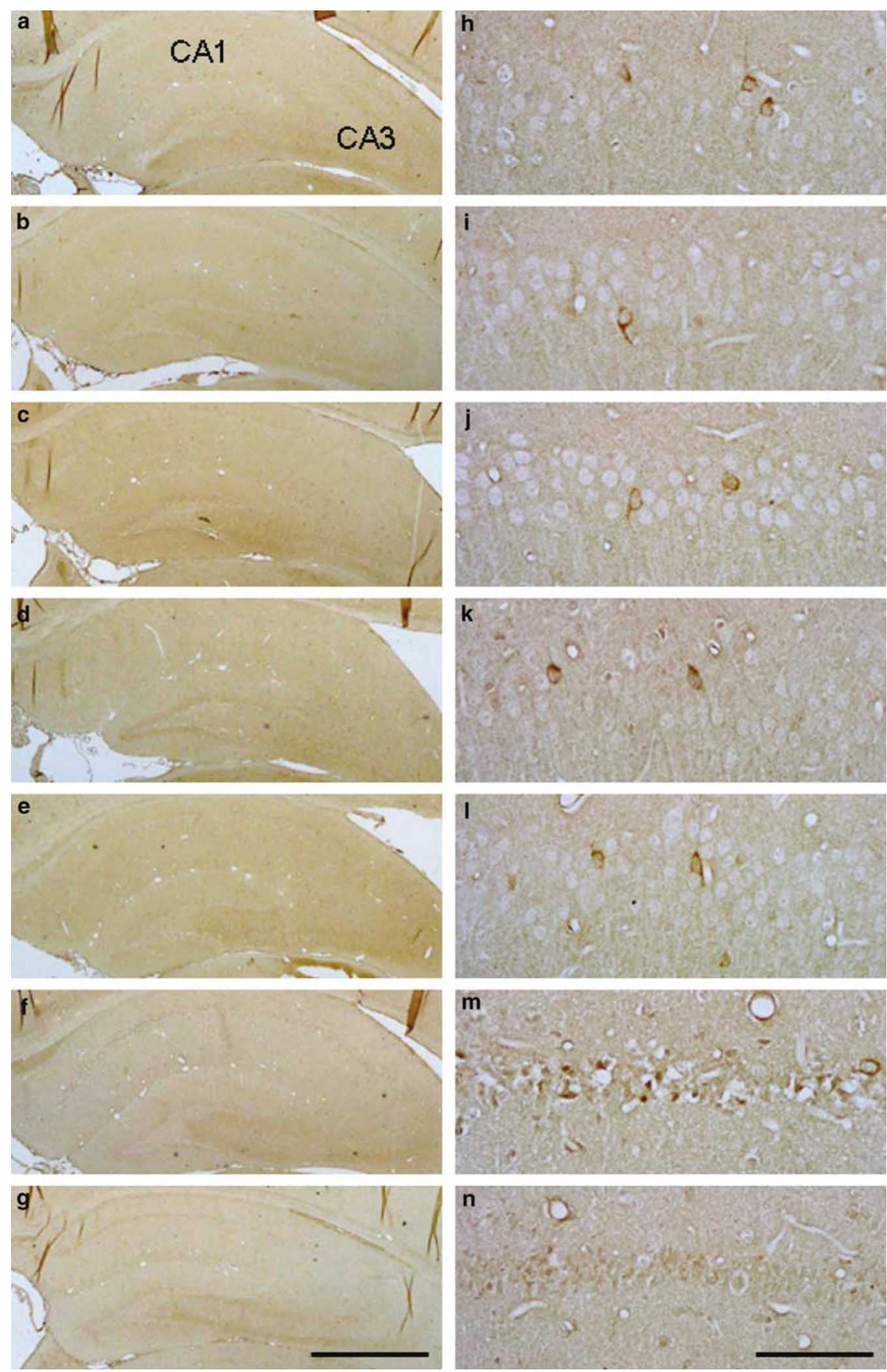

Figure 5 Representative photomicrographs with nNOS immunostaining of the hippocampus $(a-g)$ and CAI sector (h-n) after transient cerebral ischemia. $(a, h)$ : sham-operated gerbil brain. (b, i): vehicle-treated gerbil brain I h after ischemia. (c, j): vehicle-treated gerbil brain $5 \mathrm{~h}$ after ischemia. (d, $\mathrm{k}$ ): vehicletreated gerbil brain I day after ischemia. (e, l): vehicle-treated gerbil brain 2 days after ischemia. (f, $m$ ): vehicle-treated gerbil brain 5 days after ischemia. $(\mathrm{g}, \mathrm{n})$ : vehicle-treated gerbil brain 14 days after ischemia. Each group contained 5-8 animals. Bar $(\mathrm{a}-\mathrm{g})=1 \mathrm{~mm}$. Bar $(\mathrm{h}-\mathrm{n})=100 \mu \mathrm{m}$. 



Figure 6 Effect of pitavastatin on nNOS immunoreactivity in the CAI sector $\left(a^{\prime}-e^{\prime}\right)$ of the hippocampus (a-e) of the gerbil brain 5 days after transient cerebral ischemia. $\left(a, a^{\prime}\right)$ : sham-operated gerbil brain. $\left(b, b^{\prime}\right)$ : vehicle-treated gerbil brain 5 days after ischemia. $\left(c, c^{\prime}\right)$ : pitavastatin $(3 \mathrm{mg} / \mathrm{kg})$-treated gerbil brain 5 days after ischemia. $\left(\mathrm{d}, \mathrm{d}^{\prime}\right)$ : pitavastatin $(10 \mathrm{mg} / \mathrm{kg})$-treated gerbil brain 5 days after ischemia. (e, e'): pitavastatin $(30 \mathrm{mg} / \mathrm{kg})$-treated gerbil brain 5 days after ischemia. Each group contained 7-8 animals. $\operatorname{Bar}(\mathrm{a}-\mathrm{e})=1 \mathrm{~mm}$. $\operatorname{Bar}\left(\mathrm{a}^{\prime}-\mathrm{e}^{\prime}\right)=100 \mu \mathrm{m}$

et al, 1999). Furthermore, a previous study demonstrates that prophylactic treatment with HMG-CoA reductase inhibitors can decrease the severity of cerebral focal ischemic damage irrespective of serum cholesterol levels (Endres et al, 1998). We recently reported that pitavastatin can protect against the hippocampal CA1 neuronal damage after transient cerebral ischemia in gerbils (Kumagai et al, 2004; Muramatsu et al, 2004). Based on these observations, it is conceivable that the HMG-CoA reductase inhibitor pitavastatin are effective against transient cerebral ischemia.

The present study showed that prophylactic treatment with pitavastatin significantly prevented the neuronal death of the hippocampal CA1 sector 5 days after transient cerebral ischemia. Our immunohistochemical study also showed that pitavastatin prevented significant decrease of $\mathrm{PV}$-positive interneurons and nNOS-positive interneurons 



Figure 7 Representative photomicrographs with double-labeled immunostaining with PV and nNOS of the hippocampus (a) and cerebral cortex (b) in gerbils. (Red; arrow) $=P V$. (Brown; arrowhead) $=$ nNOS. $\mathrm{Bar}=50 \mu \mathrm{m}$

in the hippocampus 5 days after ischemia. We recently reported that pitavastatin can decrease the neuronal damage of the hippocampal CA1 sector after transient cerebral ischemia in gerbils via inhibition of superoxide production (Kumagai et al, 2004). Therefore, the protective effect of pitavastatin against interneurons may, at least in part, be due to its free radical scavenging activity. These results suggest that HMG-CoA reductase inhibitors can protect against the damage of interneurons in the hippocampal CA1 sector after transient cerebral ischemia, although further studies are needed to investigate the exact mechanisms for our findings.

In conclusion, the present study demonstrates that transient cerebral ischemia can cause a loss of both PV and nNOS-immunoreactive interneurons in the hippocampal CA1 sector. Our findings also show that the damage to nNOS-immunopositive interneurons may precede the neuronal cell loss in the hippocampal CA1 sector after ischemia and nNOS-positive interneurons may play some role in the pathogenesis of cerebral ischemic diseases. Furthermore, the present study suggests that transient cerebral ischemia may cause the functional damage of interneurons in the hippocampal CA3 sector. Moreover, our present study indicates that prophylactic treatment with pitavastatin can prevent the damage of interneurons in the hippocampal CA1 and CA 3 sectors after transient cerebral ischemia. Thus, our study provides valuable information for the pathogenesis after transient cerebral ischemia.

\section{ACKNOWLEDGEMENTS}

We appreciatively acknowledge Kowa Company, Ltd, Tokyo, Japan, for providing pitavastatin, and helpful advice. This study was supported in part by a Grant-in-Aid for Scientific Research (13671095 and 13670627) from the Ministry of Science and Education in Japan.

\section{REFERENCES}

Andressen C, Blumcke I, Celio MR (1993). Calcium-binding proteins: selective markers of nerve cells. Cell Tissue Res 271: 181-208.

Aoki T, Nishimura H, Nakagawa S, Kojima J, Suzuki H, Tamaki T et al (1997). Pharmacological profile of a novel synthetic inhibitor of 3-hydroxy-3-methylgluaryl-coenzyme A reductase. Arzneimittelforschung 47: 904-909.

Araki T, Kato H, Kanai Y, Kogure K (1993). Postischemic changes of intracellular second messengers in the gerbil brain after long-term survival: an autoradiographic study. Neuroscience 53: 829-836.

Araki T, Kato H, Kogure K, Kanai Y (1992). Long-term changes in gerbil brain neurotransmitter receptors following transient cerebral ischemia. Br J Pharmacol 107: 437-442.

Bouilleret V, Schwaller B, Schurmans S, Celio MR, Fritschy JM (2000). Neurodegenerative and morphogenic changes in a mouse model of temporal lobe epilepsy do not depend on the expression of the calcium-binding proteins parvalbumin, calbindin, or calretinin. Neuroscience 97: 47-58.

Bredt DS, Hwang PM, Glatt CE, Lowenstein C, Reed RR, Snyder SH (1991). Cloned and expressed nitric oxide synthase structurally resemble cytochrome $P-450$ reductase. Nature 351: 714-718.

Celio MR (1986). Parvalbumin in most $\gamma$-aminobutyric acidcontaining neurons of the rat cerebral cortex. Science 231: 995-997.

Choi DW (1993). Nitric oxide: foe or friend to the injured brain? Proc Natl Acad Sci USA 90: 9741-9743.

Dawson TM, Dawson VL (1995). Nitric oxide: actions and pathological roles. Neuroscientist 1: 7-18.

Dawson TM, Snyder SH (1994). Gases as biological messengers: nitric oxide and carbon monoxide in the brain. J Neurosci 14: 5147-5159.

Dawson VL, Dawson TM, London ED, Bredt DS, Snyder SH (1991). Nitric oxide mediates glutamate neurotoxicity in primary cortical cultures. Proc Natl Acad Sci USA 88: 6368-6371.

Dinerman JL, Dawson TM, Schell MJ, Snowman A, Snyder SH (1994). Endothelial nitric oxide synthase localized to hippocampal pyramidal cells: implications for synaptic placidity. Proc Natl Acad Sci USA 91: 4214-4218.

Endres M, Laufs U, Huang Z, Nakamura T, Huang P, Moskowitz MA et al (1998). Stroke protection by 3-hydroxy-3-methylglutaryl (HMG)-CoA reductase inhibitors mediated by endothelial nitric oxide synthase. Proc Natl Acad Sci USA 95: 8880-8885. 
Ferrer I, Lopez E, Pozas E, Ballabriga J, Marti E (1998). Multiple neurotrophic signals converge in surviving CA1 neurons of the gerbil hippocampus following transient forebrain ischemia. J Comp Neurol 394: 416-430.

Fujimoto H, Kojima J, Yamada Y, Kanda H, Kimata H (1999). Studies on the metabolic fate of pitavastatin, a new inhibitor of HMG-CoA reductase (4): interspecies variation in laboratory animals and humans. Xenobiot Metabol Dispos 14: 79-91.

Fukuda T, Nakano S, Yoshiya I, Hashimoto PH (1993). Persistent degenerative state of non-pyramidal neurons in the CA1 region of the gerbil hippocampus following transient forebrain ischemia. Neuroscience 53: 23-28.

Heizmann CW (1984). Parvalbumin, an intracellular calciumbinding protein; distribution, properties and possible roles in mammalian cells. Experimentia 40: 910-921.

Hontanilla B, Parent A, de las Heras S, Gimenez-Amaya JM (1998). Distribution of calbindin D-28k and parvalbumin neurons and fibers in the rat basal ganglia. Brain Res Bull 47: 107-116.

Huang PL, Dawson TM, Bredt DS, Snyder SH, Fishman MC (1993). Targeted disruption of the neuronal nitric oxide synthase gene. Cell 75: 1273-1286.

Iadecola C (1997). Bright and dark sides on nitric oxide in ischemic brain injury. Trends Neurosci 20: 132-139.

Iadecola C, Pelligrino DA, Moskowitz MA, Lassen NA (1994). Nitric oxide synthase inhibition and cerebrovascular regulation. J Cereb Blood Flow Metab 14: 175-192.

John S, Schlaich M, Langenfeld M, Weihprecht H, Schmitz G, Weidinger $\mathrm{G}$ et al (1998). Increased bioavailability of nitric oxide after lipid-lowering therapy in hypercholesterolemic patients: a randomized, placebo-controlled, double-blind study. Circulation 98: $211-216$.

Kaesemeyer WH, Caldwell RB, Huang J, Caldwell RW (1999). Pravastatin sodium activates endothelial nitric oxide synthase independent of its cholesterol-lowering actions. J Am Coll Cardiol 33: 234-241.

Kajinami K, Koizumi J, Ueda K, Miyamoto S, Takegoshi T, Mabuchi H (2000). Effects of NK-104, a new hydroxymethylglutaryl-coenzyme reductase inhibitor, on low-density lipoprotein cholesterol in heterozygous familial hypercholesterolemia. Hokuriku NK-104 Study Group. Am J Cardiol 85: 178-183.

Kato H, Kogure K, Araki T, Itoyama Y (1995). Graded expression of immunomolecules on activated microglia in the hippocampus following ischemia in a rat model of ischemic tolerance. Brain Res 694: 85-93.

Kirino T (1982). Delayed neuronal death in the gerbil hippocampus following ischemia. Brain Res 239: 57-69.

Kumagai R, Oki C, Muramatsu Y, Kurosaki R, Kato H, Araki T (2004). Pitavastatin, a 3-hydroxy-3-methylglutaryl-coenzyme A (HMG-CoA) reductase inhibitor, reduces hippocampal damage after transient cerebral ischemia in gerbils. J Neural Transm 111: 1103-1120.

Kurosaki R, Muramatsu Y, Kato H, Watanabe Y, Imai Y, Itoyama Y et al (2005). Effect of angiotensin-converting enzyme inhibitor perindopril on interneurons in MPTP-treated mice. Eur Neuropsychopharmacol 15: 57-67.

Kurosaki R, Muramatsu Y, Michimata M, Matsubara M, Kato H, Imai Y et al (2002). Role of nitric oxide synthase against MPTP neurotoxicity in mice. Neurol Res 24: 655-662.

Laufs U, La Fata V, Plutzky J, Liao JK (1997). Upregulation of endothelial nitric oxide synthase by HMG CoA reductase inhibitors. Circulation 97: 1129-1135.

Moncada S, Palmer RM, Higgs EA (1991). Nitric oxide: physiology, pathophysiology and pharmacology. Pharmacol Rev 43: 109-142.

Muramatsu Y, Kurosaki R, Kato H, Araki T (2004). Effect of pitavastatin against expression of $S 100 \beta$ protein in the gerbil hippocampus after transient cerebral ischemia. Acta Physiol Scand 184: 95-107.
Muramatsu Y, Kurosaki R, Mikami T, Michimata M, Matsubara M, Imai $Y$ et al (2002). Therapeutic effect of neuronal nitric oxide synthase inhibitor (7-nitroindazole) against MPTP neurotoxicity in mice. Metab Brain Dis 17: 169-182.

Muramatsu Y, Kurosaki R, Watanabe H, Michimata M, Matsubara M, Imai Y et al (2003). Expression of S100 protein is related to neuronal damage in MPTP-treated mice. Glia 42: 307-313.

Nitsch C, Scotti A, Sommacal A, Kalt G (1989). GABAergic hippocampal neurons resistant to ischemia-induced neuronal death contain the $\mathrm{Ca}^{2+}$-binding protein parvalbumin. Neurosci Lett 105: 263-268.

Noji Y, Higashikata T, Inazu A, Nohara A, Ueda K, Miyamoto S et al (2002). Long-term treatment with pitavastatin (NK-104), a new HMG-CoA reductase inhibitor, of patients with heterozygous familial hypercholesterolemia. Atherosclerosis 163: 157-164.

Paschen W (1996). Disturbances of calcium homeostasis within the endoplasmic reticulum may contribute to the development of ischemic-cell damage. Med Hypotheses 47: 283-288.

Petito CK, Feldmann E, Pulsinelli WA, Plum F (1987). Delayed hippocampal damage in humans following cardiorespiratory arrest. Neurology 37: 1281-1286.

Pulsinelli WA, Brierley JB, Plum F (1982). Temporal profile of neuronal damage in a model of transient forebrain ischemia. Ann Neurol 11: 491-498.

Schwartz-Bloom RD, Sah R (2001). $\gamma$-Aminobutyric $\operatorname{acid}_{A}$ neurotransmission and cerebral ischemia. J Neurochem 77: 353-371.

Selley ML (2005). Simvastatin prevents 1-methyl-4-phenyl-1,2, 3,6tetrahydropyridine-induced striatal dopamine depletion and protein tyrosine nitration in mice. Brain Res 1037: 1-6.

Sloviter RS, Sollas AL, Barbaro NM, Laxer KD (1991). Calciumbinding protein (calbindin-D28K) and parvalbumin immunocytochemistry in the normal and epileptic human hippocampus. J Comp Neurol 308: 381-396.

Smiley JF, McGinnis JP, Javitt DC (2000). Nitric oxide synthase interneurons in the monkey cerebral cortex are subsets of the somatostatin, neuropeptide Y, and calbindin cells. Brain Res 863: 205-212.

Solodkin A, Veldhuizen SD, Van Hoesen GW (1996). Contingent vulnerability of entorhinal parvalbumin-containing neurons in Alzheimer's disease. J Neurosci 16: 3311-3321.

Suzuki H, Aoki T, Tamaki T, Sato F, Kitahara M, Saito Y (1999). Hypolipidemic effect of NK-104, a potent HMG-CoA reductase inhibitor, in guinea pigs. Atherosclerosis 146: 259-270.

Tortosa A, Ferrer I (1993). Parvalbumin immunoreactivity in the hippocampus of the gerbil after transient forebrain ischemia: a qualitative and quantitative sequential study. Neuroscience 55: 33-43.

Valtschanoff JG, Weinberg RJ, Kharazia VN, Schmidt HH, Nakane M, Rustioni A (1993). Neurons in rat cerebral cortex that synthesize nitric oxide: NADPH diaphorase histochemistry, NOS immunocytochemistry, and colocalization with GABA. Neurosci Lett 157: 157-161.

Van Den Bosch L, Schwaller B, Vleminckx V, Meijers B, Stork S, Ruehlicke $\mathrm{T}$ et al (2002). Protective effect of parvalbumin on excitotoxic motor neuron death. Exp Neurol 174: 150-161.

Vincent SR, Kimura H (1992). Histochemical mapping of nitric oxide synthase in the rat brain. Neuroscience 46: 755-784.

Yun HY, Dawson VL, Dawson TM (1997). Nitric oxide in health and disease of the nervous system. Mol Psychiatry 2: 300-310.

Zucker B, Luthi-Carter R, Kama JA, Cunah AW, Stern EA, Fox JH et al (2005). Transcriptional dysregulation in striatal projectionand interneurons in a mouse model of Huntington's disease: neuronal selectivity and potential neuroprotective role of HAP1. Hum Mol Genet 14: 179-189. 